\title{
STROKE
}

\section{Improved lesion-symptom mapping in poststroke aphasia}

A new study has uncovered distinct phenotypes of poststroke aphasia related to unique patterns of damage in the left hemisphere.

Aphasia is common after middle cerebral artery stroke, but patients can display remarkable heterogeneity in their difficulties with the production or comprehension of language. "Aphasia is not a monolithic diagnostic category," explains study investigator Matthew Lambon Ralph. "Instead, there are various distinct subtypes of aphasia, and graded differences between them."

Conventional lesion-mapping techniques have difficulty isolating links between damaged areas and specific deficits. This shortcoming might be related to the fact that these maps do not account for both damage severity and regionality: many areas will be affected by stroke, but not all areas will contribute to aphasia.

Lambon Ralph, together with Anna Woollams and Rebecca Butler, adopted a multifaceted approach to dissociate aphasia phenotypes. The investigators recruited 31 participants with aphasia after left hemisphere stroke, and 19 matched controls. Participants then took a battery of neuropsychological tests to assess linguistic function.

The researchers used principal component analysis to uncover the main variables associated with aphasia symptoms, which revealed three independent components to the patients' performance. These domains were phonology, for interpreting and producing sounds; semantics, for judging word meaning; and executive cognition, for solving problems and changing strategies.

Lesions were mapped via an automated MRI processing procedure. "When we used individual tests or patient classifications to correlate with lesion distributions, we found

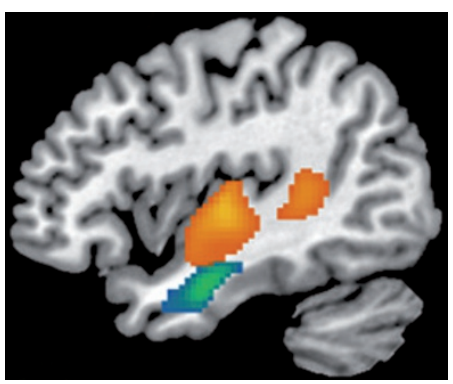

Language processing in poststroke aphasia: orange indicates areas linked to phonology and blue and green indicate areas linked to semantics. Image courtesy of Anna Woollams.

the typical pattern of large, overlapping areas of damage," says Lambon Ralph. The investigators therefore applied a voxel-based correlational methodology to generate regression coefficients linking areas in the left hemisphere to each of the aphasia components.

Phonological deficits were associated with damage to perisylvian regions of cortex and white matter projections beneath the posterior superior temporal gyrus. Semantic impairments were associated with lesions focused on the anterior middle temporal gyrus and the temporal stem. No unique areas were isolated for executive deficits.

The results shed further light on the brain's language networks, and Lambon Ralph highlights the consistency with the notion of dorsal and ventral language pathways. Moreover, the work might pave the way for improved assessment of patients after stroke.

"It might be possible to use scans from early after stroke as predictors of later language status and type of language problem," concludes Lambon Ralph. This information could then be used to tailor management and treatment strategies to ensure the best possible outcome.

\section{Alex Chase}

Original article Butler, R. et al. Capturing multidimensionality in stroke aphasia: mapping principal behavioural components to neural structures. Brain doi:10.1093/brain/awu286 